\title{
Uroguanylin: how the gut got another satiety hormone
}

\author{
Randy J. Seeley and Matthias H. Tschöp
}

Metabolic Diseases Institute, Department of Internal Medicine, University of Cincinnati, Cincinnati, Ohio, USA.

\begin{abstract}
Prouroguanylin is a gastrointestinal paracrine signal and prohormone that is secreted after nutrient ingestion. In this issue of the JCI, Valentino et al. show that prouroguanylin is converted to uroguanylin in the CNS, which can activate guanylyl cyclase $2 \mathrm{C}$ (GUCY2C) receptors in the brain to reduce food intake in mice. This 16 -amino acid residue peptide is a novel component of the gut-brain axis that represents a new and unique opportunity to manipulate gut-brain signaling for therapeutic intervention in obesity.
\end{abstract}

Life as an endocrinologist is getting complicated these days. A few decades ago, there were just a small number of endocrine organs, including the thyroid, adrenals, and pancreas. Over the past 20 years, the list of organs that are considered major endocrine organs has expanded to include adipose tissue, liver, bone, and muscle, among others. The endocrine productivity of these organs, however, cannot compete with that of the gastrointestinal (GI) tract. From the stomach to the rectum, there are numerous distinct cell populations that secrete a wide range of hormones involved in a diverse array of functions (1).

One of the key functions of GI hormones is the regulation of food intake and the distribution of ingested nutrients (2). Particularly intriguing data highlighting the important role of GI hormones in this context come from the assessment of patients undergoing bariatric surgery, for whom a variety of manipulations of the GI tract result in profound weight loss and the resolution of comorbid conditions, such as type 2 diabetes (3). While it remains a possibility that the changes that result from bariatric surgery are the direct result of the physical restriction to food intake and nutrient absorption and/or malabsorption that may accompany such procedures, it has become

Conflict of interest: Randy J. Seeley has received consulting and speaking fees from Novo Nordisk, Merck, and Ethicon Endo-Surgery. Randy J. Seeley's laboratory has been supported by research funds from Ethicon Endo-Surgery, Novo Nordisk, MannKind Corp., Pfizer, and Ablaris Therapeutics Inc. Matthias H. Tschöp has received consulting and speaking fees from Marcadia Biotech, Roche, Merck, and Ambrx. Matthias H. Tschöp's laboratory has been supported by Ambrx, Marcadia Biotech, Roche, and Ethicon Endo-Surgery.

Citation for this article: J Clin Invest. 2011; 121(9):3384-3386. doi:10.1172/JCI58297. increasingly accepted that modulation of the levels of a wide range of factors secreted by the GI tract may have a crucial role in the effectiveness of these therapies (4).

In the clinical arena, GI hormones have a potential role as the molecular signals that mediate the metabolic benefits of bariatric surgeries, and several new GI hormone-based drugs are now available to the patient or are in clinical testing for the treatment of metabolic diseases. A key role for the GI tract, as both potential cause and cure for escalating levels of metabolic diseases therefore seems likely, making a thorough understanding of its complex endocrine functions critical for our success in the fight against obesity and type 2 diabetes. In this issue of the JCI, Valentino et al. report a new player involved in the cornucopia of hormones that is the GI endocrine system (5). Identifying this novel system opens up new possibilities for GI-based therapies for helping obese individuals lose weight.

\section{Guanylin and uroguanylin: new gut hormones}

Guanylin and uroguanylin are peptides of 15 - and 16-amino acids in length, respectively. They are both secreted by intestinal epithelial cells as prohormones, which require enzymatic conversion into active hormones. To date, their function has been thought to be largely paracrine. Upon secretion into the lumen of the GI tract, they act on guanylyl cyclase 2C (GUCY2C) receptors on intestinal epithelial cells, increasing intracellular cyclic GMP (cGMP). Interestingly, GUCY2C receptors also are the target for heat-stable enterotoxins and upon activation can decrease intestinal fluid absorption, which can lead to diarrhea $(6,7)$.
cGMP, however, has also been implicated in the regulation of nutrient intake in invertebrate models (8). Therefore, Valentino and colleagues hypothesized that uroguanylin or guanylin might act as part of the gut-brain axis that regulates ingestion, energy homeostasis, and body weight (5). Consistent with this hypothesis, they found that mice engineered to globally lack the GUCY2C receptor carried more body fat as a direct result of increased food intake. These observations were further corroborated when they observed that systemic administration of a GUCY2C agonist reduced food intake in wild-type mice but not GUC2YC-deficient mice.

\section{Prouroguanylin: a postprandial hormone that is activated in the brain}

One of the key questions after having determined that signaling via the GUCY2C receptor regulates ingestion, energy homeostasis, and body weight was what is the identity of the endogenous ligand for GUCY2C that represents a novel satiety factor? Valentino et al. found that the precursor to uroguanylin, prouroguanylin, is secreted after meals in both mice and humans (5). However, this left an important problem: prouroguanylin does not activate GUCY2C, it must be cleaved into uroguanylin, which then can activate that receptor. Where might this occur to result in satiation?

In a very clever experiment, Valentino et al. found that when either prouroguanylin or proguanylin were administered to a cell line expressing GUCY2C receptors, no increase in cGMP levels was observed (5). However, if prouroguanylin (but not proguanylin) was mixed with protein extracts from hypothalamus tissue, cGMP levels could be elevated. These experiments provided strong evidence to suggest that the hypothalamus is capable of converting prouroguanylin into active uroguanylin. Consistent with this conclusion, Valentino et al. found that GUCY2C receptors were expressed in the hypothalamus and that administration of a GUCY2C agonist directly into the CNS 


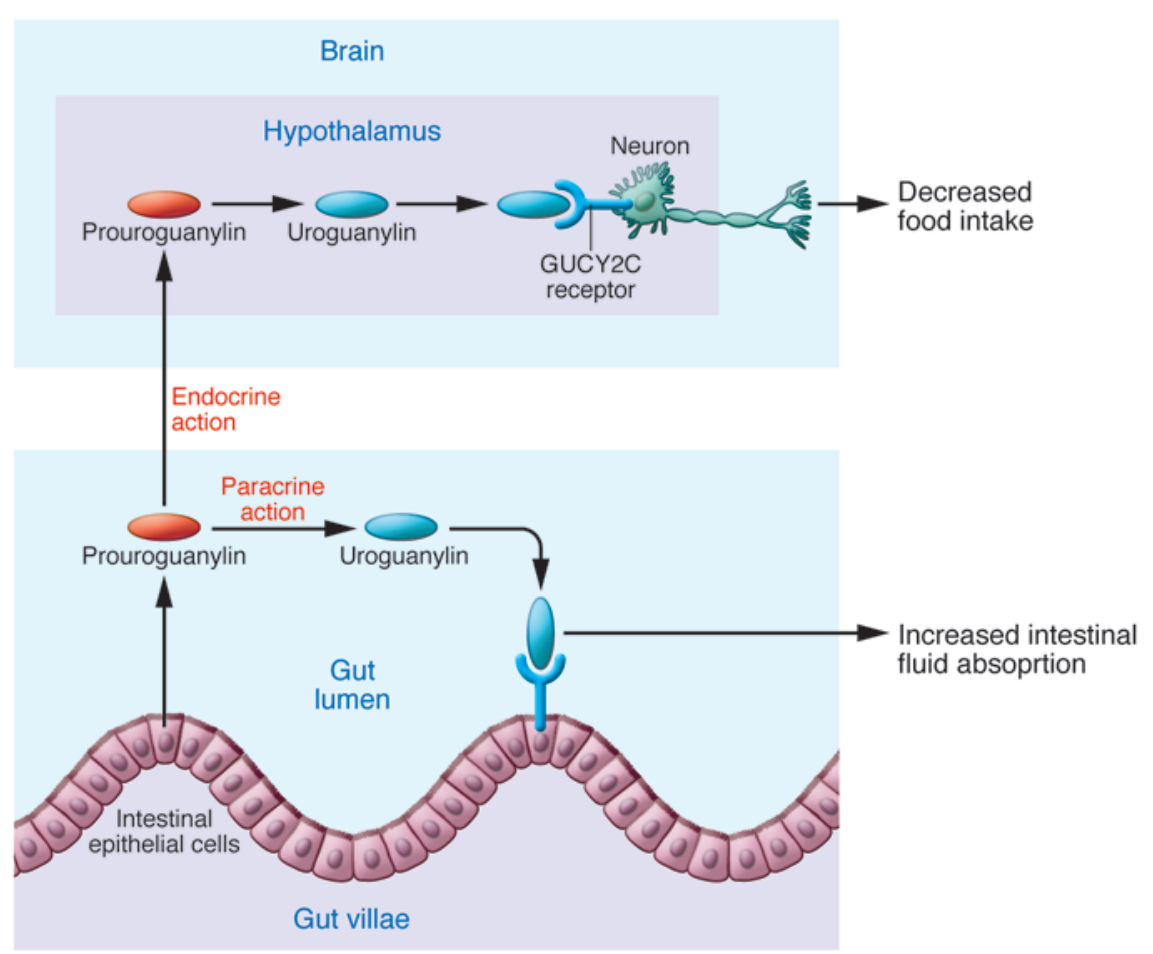

resulted in a reduction of food intake (5), providing tangible proof for a unique new component of the gut-brain signaling axis that controls metabolic homeostasis.

\section{Uroguanylin: a GI hormone different than others?}

Several GI hormones depend on unique posttranslational modifications as critical determinants of their actions. For example, glucagon-like peptide 1 (GLP-1) and glucose-dependent insulinotropic polypeptide (GIP) have a very short half-life, due to inactivation by the relatively ubiquitous protease dipeptidyl-peptidase 4 (DPP-4) (9). On the flip side of the DPP-4 equation is peptide YY (PYY). PYY is secreted as a 36-amino acid residue peptide and rapidly cleaved by DPP-4 into PYY3-36, which has been reported to play a role in the neuroendocrine control of food intake (10). The primarily stomachderived hormone ghrelin requires the addition of a fatty acid side chain to activate its known receptor and alter food intake (11). This modification appears to happen in the same GI cells that synthesize the ghrelin peptide prior to its release.

The discovery of uroguanylin as a satiety factor (5) adds yet another posttranslational strategy that the GI tract uses to send afferent signals to the brain in order to maintain metabolic homeostasis. After meals, the hormonally inactive propeptide (prouroguanylin) appears to be secreted and transported via blood circulation to the CNS, in which it is converted to the active hormone, activates its cognate receptor, and suppresses food intake (Figure 1). This discovery impressively illuminates the many possibilities evolution has generated by which gut signals control systemic energy metabolism beyond classic endocrine mechanisms, such as modulating hormone secretion and receptor expression. It implies that, for reasons that are not obvious, it was advantageous for survival to find multiple alternative ways to fine tune the potency and the kinetics by which a complex array of endocrine signals could control energy balance.

\section{A road to new therapies?}

The bottom line of the work of Valentino et al. (5) is that it looks like uroguanylin should be added to the long list of hormones that appear to be part of how the gut convinces us to put our forks down and end a meal. A number of important questions will have to be answered by future studies, including whether the here reported functions of this hormone system are relevant in humans. It would also be important to find out whether there are particular nutrients that specifically trigger this pathway and whether its actions are modulated by bariatric surgery. Given the unique biol-

\section{Figure 1}

Prouroguanylin is a prohormone that is secreted by the GI tract. It has been shown to be cleaved in the lumen of the GI tract to the active hormone, uroguanylin, that can activate GUCY2C receptors to alter fluid absorption. In this issue of the $\mathrm{JCl}$, Valentino et al. (5) show that prouroguanylin levels in the blood are increased after meals. It can then be cleaved to uroguanylin in the CNS to interact with GUCY2C receptors in the hypothalamus to decrease food intake and thereby act as a satiety factor from gut to brain. ogy of uroguanylin, it may offer a number of potential possibilities for therapeutic intervention to help obese individuals lose weight. GUCY2C agonists could be developed that would suppress food intake, and one could design pharmacological strategies to increase the secretion of prouroguanylin or promote the conversion of the prohormone into uroguanylin within the key target regions in the CNS. Finally, we could take a page out of the uroguanylin playbook we have just been given by making prodrugs that are cleaved into GUCY2C agonists by the same enzymes that cleave prouroguanylin. Such a strategy would provide a method to directly deliver the drug to the key target tissue, while hopefully limiting the potential side effects associated with activating GUCY2C receptors in the GI tract. Successful targeting of this pathway for the development of novel therapeutics for obesity would likely depend to a considerable extent on the ability of the therapeutics to divorce the beneficial effects in the hypothalamus from an undesired impact on intestinal fluid absorption.

While the results of Valentino et al. (5) are extremely exciting and clinically significant, it is still too soon to declare victory in the fight to limit the obesity epidemic. There are several examples in which intriguing reports on novel peptide processing seemed to promise rapid breakthroughs 
for the therapy of metabolic diseases but never delivered. In 2005, the "hormone" obestatin was reported as a previously overlooked by-product of preproghrelin cleavage with the ability to induce satiety in the CNS (12). Since then, scientists in the field have struggled to repeat the effects of obestatin on food intake or body weight seen in the previous report as well as to confirm its ability to bind and activate G protein-coupled receptor 39 (12).

With all of the GI hormones identified to date, it has turned out that their actions in the CNS are neither restricted to receptor activation in a single brain region nor limited to the modulation of a single physiological function. GLP-1 receptors, for example, are widely distributed throughout the brain and have been linked to visceral illness, anxiety, glucocorticoid secretion, and even promotion of neuronal survival (13, 14). As another example, ghrelin appears to act in key regions of the hippocampus, olfactory cortex, and ventral tegmental area to regulate memory, reward functions, and even mood (15-17), in addition to affecting energy balance in the hypothalamus. So while the data generated by Valentino et al. (5) point to a discrete satiety-inducing function for uroguanylin within the hypothalamus, it seems likely that it will act at multiple target sites and serve a wider range of yet to be determined physiological processes. Whether these will limit or enhance the therapeutic potential of this intriguing system awaits further study. However, with this important discovery by Valentino et al. (5), yet another potential tool has been provided to harness the biology of the gut-brain axis to help fill the medical need to treat obesity and diabetes.

\section{Acknowledgments}

Randy J. Seeley was supported by NIDDK DK54890. The authors would like to acknowledge J. Walker for facilitating discussions related to this commentary.

Address correspondence to: Randy J. Seeley, Metabolic Diseases Institute, 2170 E. Galbraith Rd., University of Cincinnati, Cincinnati, Ohio 45237, USA. Phone: 513.558.6665; Fax: 513.558.0187; E-mail: randy.seeley@uc.edu.

1. Wren AM. Gut and hormones and obesity. Front Horm Res. 2008;36:165-181.

2. Strader AD, Woods SC. Gastrointestinal hormones and food intake. Gastroenterology. 2005; 128(1):175-191.

3. Buchwald $\mathrm{H}$, et al. Weight and type 2 diabetes after bariatric surgery: systematic review and meta-analysis. Am J Med. 2009;122(3):248-256.

4. Thaler JP, Cummings DE. Minireview: Hormonal and metabolic mechanisms of diabetes remission after gastrointestinal surgery. Endocrinology. 2009;150(6):2518-2525.
5. Valentino MA, et al. A uroguanylin-GUCY2C endocrine axis regulates feeding in mice. $J$ Clin Invest. 2011;121(9):3578-3588.

6. Sindic A, Schlatter E. Renal electrolyte effects of guanylin and uroguanylin. Curr Opin Nephrol Hypertens. 2007;16(1):10-15.

7. Forte LR Jr. Uroguanylin and guanylin peptides: pharmacology and experimental therapeutics. Pharmacol Ther. 2004;104(2):137-162.

8. You YJ, Kim J, Raizen DM, Avery L. Insulin, cGMP, and TGF-beta signals regulate food intake and quiescence in C. elegans: a model for satiety. Cell Metab. 2008;7(3):249-257.

9. Drucker DJ. The biology of incretin hormones. Cell Metab. 2006;3(3):153-165

10. Renshaw D, Batterham RL. Peptide YY: a potential therapy for obesity. Curr Drug Targets. 2005; 6(2):171-179.

11. Kirchner $\mathrm{H}$, et al. GOAT links dietary lipids with the endocrine control of energy balance. Nat Med. 2009;15(7):741-745

12. Zhang JV, et al. Obestatin, a peptide encoded by the ghrelin gene, opposes ghrelin's effects on food intake. Science. 2005;310(5750):996-999.

13. Kinzig KP, et al. CNS glucagon-like peptide-1 receptors mediate endocrine and anxiety responses to interoceptive and psychogenic stressors. J Neurosci. 2003;23(15):6163-6170.

14. Kinzig KP, D'Alessio DA, Seeley RJ. The diverse roles of specific GLP-1 receptors in the control of food intake and the response to visceral illness. J Neurosci. 2002;22(23):10470-10476.

15. Chuang JC, Zigman JM. Ghrelin's roles in stress, mood, and anxiety regulation. Int J Pept. 2010; 2010:460549.

16. Lutter M, et al. The orexigenic hormone ghrelin defends against depressive symptoms of chronic stress. Nat Neurosci. 2008;11(7):752-753.

17. Tong J, et al. Ghrelin enhances olfactory sensitivity and exploratory sniffing in rodents and humans. J Neurosci. 2011;31(15):5841-5846

\title{
Behind an enteric neuron there may lie a glial cell
}

\author{
Michael D. Gershon
}

Department of Pathology and Cell Biology, Columbia University, College of Physicians and Surgeons, New York, New York, USA.

\begin{abstract}
The enteric nervous system (ENS) controls the gastrointestinal system. Enteric glia have long been regarded as the essential "glue" of the ENS. Now, however, two independent reports in this issue of the JCI provide compelling evidence that mouse enteric glia can also be neuronal precursors. These reports show that enteric glia give rise to neurons in vitro and that neurogenesis can be experimentally induced to occur in vivo in the adult mouse ENS. Unfortunately, glia do not constitutively replace neurons, and neurogenesis is not easily provoked. Although these new observations make it clear that clinical trials using glia to replace enteric neurons are more than premature, they are enticing for future research.
\end{abstract}

The enteric nervous system (ENS) is a neural crest-derived division of the autonomic nervous system that is essential for life (1).

Conflict of interest: The author has declared that no conflict of interest exists.

Citation for this article: J Clin Invest. 2011; 121(9):3386-3389. doi:10.1172/JCI59573.
It controls the gastrointestinal (GI) system and comprises a large number of neurons and glia that are organized into complex networks of interconnected ganglia distributed throughout the gut wall. Enteric neurons cluster into two plexi: the myenteric plexus, which is situated between the inner circular and outer longitudinal layers of the muscularis externa; and the submucosal plexus, which is located within the dense connective tissue between the muscularis externa and the mucosa.

One of the many functions of the GI system controlled by the ENS is motility, and thereby GI transit. As a result, aganglionosis of the bowel, whether congenital (as in Hirschsprung disease) or acquired (as in Chagas disease), leads to intestinal obstruction $(2,3)$. Nerve bundles, however, are present in the aganglionic segments of colon in individuals with Hirschsprung disease, meaning that nerves are not, by themselves, sufficient for GI transit (4). Nerve cell bodies and the complex microcircuits of the ENS, which uniquely enable it to control GI motility and secretion in the absence of CNS input, are required for normal GI tran- 